\title{
Analysis of liver fragment subjected to autologous transplant at rat's retroperitoneum
}

\author{
Mirla Fiuza Diniz, BSc, Sávio Lana Siqueira, PhD,* \\ Tiago Soares Baumfeld, MD, Luis Felipe Cintra Pereira, MD, \\ Filipe Garcia Moreira, MD, Gustavo Meirelles Ribeiro, MD, \\ and Iure Kalinine Ferraz de Souza, PhD
}

School of Medicine, Federal University of Ouro Preto (UFOP), Ouro Preto, Brazil

\section{A R T I C L E I N F O}

Article history:

Received 14 January 2015

Received in revised form

22 May 2015

Accepted 11 June 2015

Available online 17 June 2015

Keywords:

Hepatic regeneration

Autotransplantation

Experimental surgery

\begin{abstract}
A B S T R A C T
Background: To investigate the regeneration process of autologous implants of liver on the retroperitoneum.

Methods: Thirty male Fisher rats were used divided in to group 1 (G1): studied $60 \mathrm{~d}$ after surgery; group 2 (G2): studied 90 d after surgery; group 3 (G3): studied $180 \mathrm{~d}$ after surgery; and group C (GC): animals without surgery. Hepatic fragment was processed for histologic and biochemical analysis.

Results: There was inflammatory infiltrate, diffuse hydropic degeneration, necrosis, and moderate fibrosis that reduced in direct relation to the postsurgical time. The concentration of albumin was different between GC and $G 1$ and between $G 1$ and $G 3(P=0.0007)$. The Catalase (CAT) was related to the time of surgery with GC being different when compared with G1, G2, and G3 $(P<0.0001)$. The oxidative stress measured through the thiobarbituric acid reactive substances lipid peroxidation was different between the GC and the G2 groups $(P=0.0381)$. Conclusions: The analysis made showed hepatic regeneration in the fragment subjected to autologous transplant at the retroperitoneum.
\end{abstract}

(c) 2015 Elsevier Inc. All rights reserved.

\section{Introduction}

Since its first realization [1], organ transplant revolutionized medical history and saved thousands of patients. An example of that is liver transplant, which drastically improved prognosis, morbidity, and mortality of patients with terminal stage hepatic disease [2]. In the last $50 \mathrm{y}$, transplant has been the only treatment for patients with terminal stage hepatic disease [3].

Liver transplant has many complications, especially when it is from a living donor [4]. The most frequent complications are those related to transplant rejection and to the biliary tract. Of transplant patients, $10 \%-40 \%$ develop biliary complications associated with a mortality rate of $8 \%-15 \%$ $[5,6]$. To alleviate the high demand for liver transplants, the interest in liver cell therapy has been increasing continuously in recent years [7].

The necessity of intervening in the evolution of tumors and/or chronic diseases, especially those related to the hematopoietic system, led to a new therapy, the autologous transplant [8]. To avoid homologous transplant complications, among other benefits, studies on hepatic regeneration are necessary $[9,10]$. Thus, our aim was to investigate the regeneration on autologous transplant of liver tissue on rat's retroperitoneum, evaluating the viability and functionality of selftransplanted hepatic tissue.

\footnotetext{
* Corresponding author. Campus Morro do Cruzeiro, School of Medicine, Federal University of Ouro Preto (UFOP), Ouro Preto 35400 000, Brazil. Tel./fax: +5503199837286.

E-mail address: saviolanasiqueira@gmail.com (S.L. Siqueira). 0022-4804/\$ - see front matter (c) 2015 Elsevier Inc. All rights reserved.
} 


\section{Materials and methods}

\subsection{Animals}

Animal care and experimental procedures were approved by the Ethics Committee on Animal Use at the Federal University of Ouro Preto (UFOP), under protocol number 2010/047, and followed the rules established by the Brazilian Society of Laboratory Animal Science. During all experiments, animals were kept at room temperature $\left(21^{\circ} \mathrm{C} \pm 2^{\circ} \mathrm{C}\right)$ and under controlled light cycles at the vivarium of the School of Nutrition (UFOP), with food and drinking water ad libitum. Thirty Fisher rats, of the species Rattus norvagius, were used, all male, with an approximate weight of $350 \mathrm{~g}$ and an approximate age of $90 \mathrm{~d}$. The rats were divided into three groups of 10 animals each: group 1 (G1) was composed of animals that were studied $60 \mathrm{~d}$ after surgery; group 2 (G2) was composed of animals that were studied after 90 d after surgery; group 3 (G3) was composed of animals that were studied $180 \mathrm{~d}$ after surgery. Group C (GC) was composed of five control animals.

\subsection{Surgical procedure}

Under an anesthesia of ketamine $(80 \mathrm{mg} / \mathrm{kg}$ ) and xylazine ( $7 \mathrm{mg} / \mathrm{kg}$ ), the animal, after trichotomy and antisepsis, was subjected to a median supraumbilical longitudinal laparotomy of about $3-4 \mathrm{~cm}$ long, extending to the xiphoid process. It was then made the mobilization of the liver's left lobe, so the selection of the fragment $(5 \mathrm{~mm} \times 5 \mathrm{~mm}$ ) to be biopsied could be made. After a simple stitch repair with a catgut 4-0 suture, at the vascular root of the segment, a wedge incision in the ischemic portion (triangular, with the base toward the hepatic edge) was made, observing a complete excision of it. Right after that, starting from the repair stitch, a Greek running suture was made to get the liver's edges closer together. The retroperitoneum was exposed, with the externalization of the animal's intestines. After visualization of the right kidney, a small incision in the retroperitoneum (root of the mesentery) was made, near the kidney's lower pole. The piece, already treated, was placed through the surgical orifice and a running suture was made in the retroperitoneum with a catgut 40 thread. At this stage, the anatomic structures were carefully observed, due to the delicacy of the organs and of the renal capsule, as well as to the possibility of ligature of the renal hilum and of the ureter.

\subsection{Euthanasia and tissue collection}

The animals' euthanasia occurred by overdose of anesthetics with sodium pentobarbital (100 mg/kg, intramuscular), after the respective postsurgery time of each group. After the administration of the anesthetic, animals were perfused with $0.9 \%$ saline solution, and the liver's right lobe, of the control animals, and the hepatic fragment selftransplanted (mean weight was $70 \pm 5.2 \mathrm{mg}$ ) were collected from animals submitted surgery. The organs were each divided into two samples. The first sample was fixed in buffered formalin solution for at least $48 \mathrm{~h}$ as to be used in the histologic analysis, and the second sample was immediately frozen at $-80^{\circ} \mathrm{C}$ as to be used in the biochemical analysis.

\subsection{Histologic staining and morphometric analysis}

After fixation, the tissue samples of the liver's right lobe and the hepatic fragment selftransplanted were processed according to routine histologic techniques and embedded in paraffin blocks. Paraffin blocks were sectioned using a microtome into $4-\mu \mathrm{m}$-thick sections, placed onto glass slides, and fixed for histologic staining. Staining was performed using hematoxylin-eosin and Masson trichrome stains.

All morphometric analyses were performed at the Multiuser Laboratory of the Research Center for Biological Sciences, Federal University of Ouro Preto. To count the number of inflammatory cells present in the hepatic lobes, measure the different liver areas, quantify the collagen fibers and the glycogen deposition, and determine the hepatic capsule thickness; we randomly obtained 20 images from histologic slides that were prepared from the liver sections. These slides were scanned using the Leica Application software and analyzed using the Leica Q-Win Plus software (Leica Microsystems, Inc, Buffalo Grove, IL) [11].

\subsection{Biochemical analysis}

The liver homogenates were used to assess the tissue-specific activities of antioxidant defense enzymes and oxidative damage, comparing the transplanted liver biopsy with liver fragments in situ. Catalase (CAT) activity was measured by the ability to convert hydrogen peroxide into water and molecular oxygen and detected as a decrease in absorbance at $240 \mathrm{~nm}$ [12]. The total protein content of the samples was verified by the Lowry technique [13]. The thiobarbituric acid reactive substances (TBARS) assays to quantify malondialdehyde levels were performed by heating the samples in the presence of thiobarbituric acid and measuring the absorbance of the supernatant at $532 \mathrm{~nm}$ [14]. Albumin was measured by immunonephelometry at $630 \mathrm{~nm}$ [15].

\subsection{Statistical analysis}

The descriptive statistical analysis and inferences were made by the Prism 5.0 software (GraphPad Software, Inc, La Jolla, CA). All data were represented with mean and standard deviation, being the evaluation of the data parametric distribution made with the normality test. For comparison of the three groups, the analysis of variance was used for parametric samples and the Kruskal-Wallis one-way analysis of variance for nonparametric data, followed by post hoc Dunn test. The statistical significance was $5 \%(P<0.05)$.

\section{Results}

\subsection{Histologic analysis}

The histologic analysis was based on four parameters that evidence the process of hepatic regeneration: inflammation, 


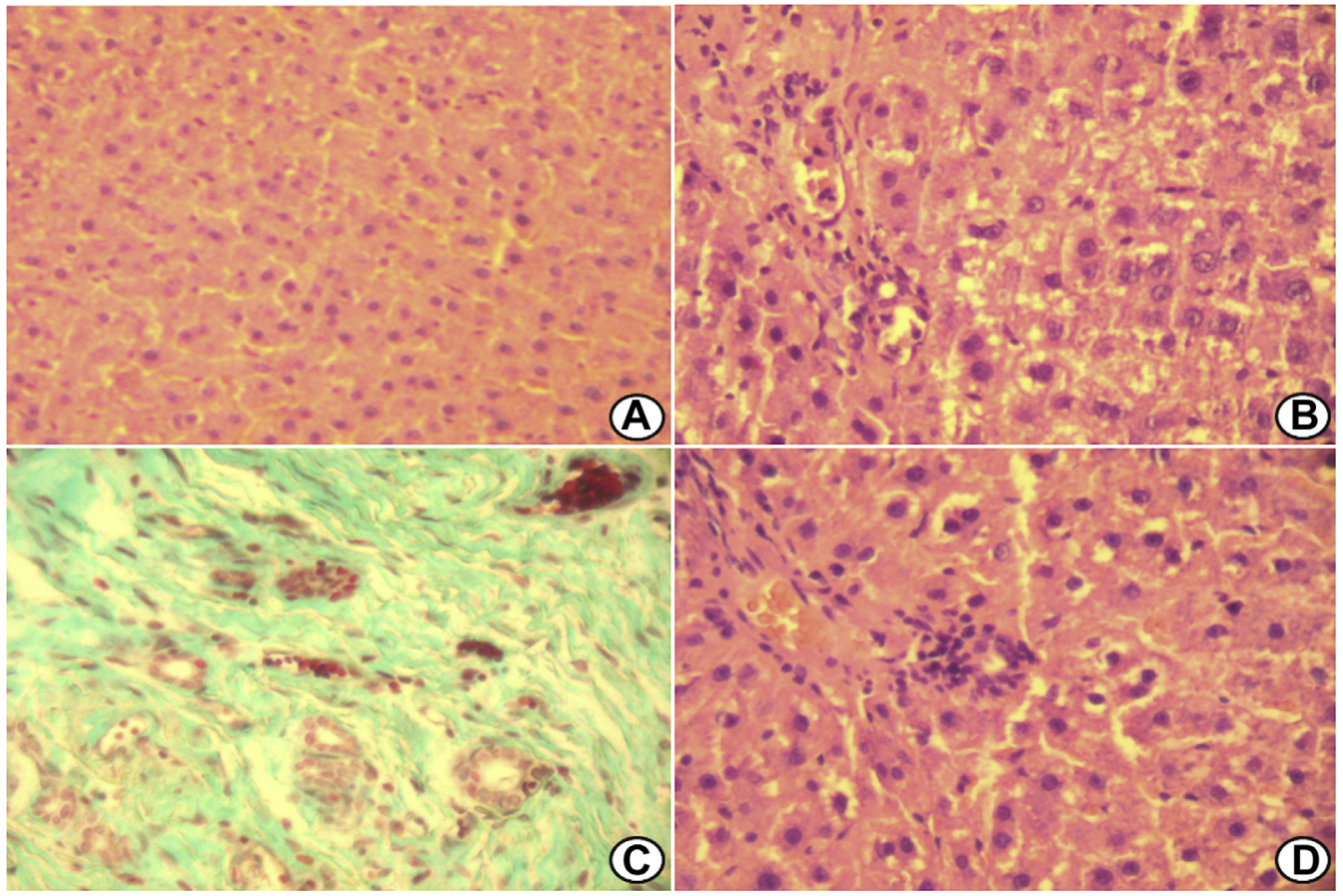

Fig. 1 - Photomicrographs of liver fragments implanted in the retroperitoneum. (A) Normal liver tissue (CG)-hematoxylin and eosin. (B) Diffuse hydropic degeneration with mononuclear inflammatory infiltrate in the periportal tracts and liver parenchyma (G1) - hematoxylin and eosin. (C) Fibrosis, neovascularization, remaining ducts, and mild inflammatory infiltrate (G2)-Masson trichrome. (D) Mild hydropic degeneration with mononuclear inflammatory infiltrate in the periportal tracts (G3)-hematoxylin and eosin. Group 1 (G1): studied $60 \mathrm{~d}$ after surgery; group 2 (G2): studied $90 \mathrm{~d}$ after surgery; group 3 (G3): studied $180 \mathrm{~d}$ after surgery; and group C (GC): animals without surgery. (Color version of figure is available online.)

degeneration, necrosis, and fibrosis on the hepatic fragment. The tissue near the fragment was also evaluated (Fig. 1).

The hepatic fragments of the first group (G1), removed $60 \mathrm{~d}$ after surgery, presented mononuclear inflammatory infiltrate, diffuse hydropic degeneration, moderate necrosis, and moderate fibrosis. Those are evidence of alterations in the hepatocytes, especially after the loss of vascularization, when the liver fragment was removed and debrided before being inserted in the retroperitoneum.

Hepatic fragments of group 2 (G2), collected $90 \mathrm{~d}$ after surgery, presented hydropic degeneration but no necrosis or fibrosis and minimal inflammation. It is believed that after a longer time of transplant (90 d), the fragments recovered their activity and started regenerating.

The group 3 (G3) samples, removed $180 \mathrm{~d}$ after surgery, showed results similar to those of G2, with both samples not having inflammatory infiltrate, degeneration, necrosis, nor fibrosis, and the hepatic fragment being integrate, with no significant alteration, presenting only a discrete mononuclear inflammatory infiltrate in the portal tracts.

The quantification of inflammatory cells in the retroperitoneal implant showed a gradual reduction according to length of permanence of the fragment, indicating the restructuring of the heterotopic hepatic parenchyma; all the three groups showed statistic difference compared with that of GC $(P<0.0001 ; 15.60 \pm 3.36 \times 74.60 \pm 4.09 \times 64.20 \pm 2.68 \times$ $53.20 \pm 2.77 \mathrm{~g} / \mathrm{dL} ; \mathrm{GC}, \mathrm{G} 1, \mathrm{G} 2$, and G3, respectively, Fig. 2).

\subsection{Biochemical analysis}

The albumin concentration was significantly different between the GC and the G1, and between the G1 and the G3 groups $(P=0.0007)$, increasing proportionally to the

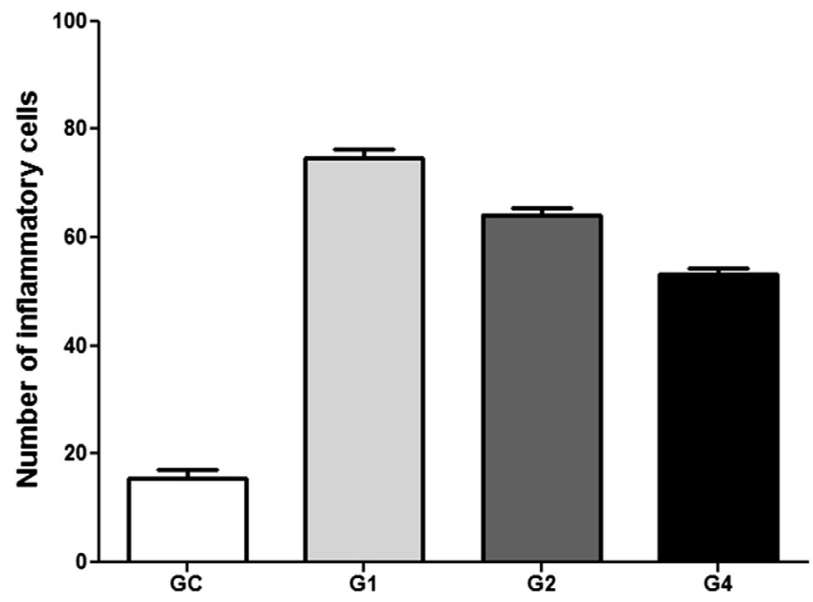

Fig. 2 - Quantification of inflammatory cells of the heterotopic fragment hepatic showed a gradual reduction according to length of permanence of the fragment. Group 1 (G1): studied $60 \mathrm{~d}$ after surgery; group 2 (G2): studied $90 \mathrm{~d}$ after surgery; group 3 (G3): studied $180 \mathrm{~d}$ after surgery; and group C (GC): animals without surgery. 

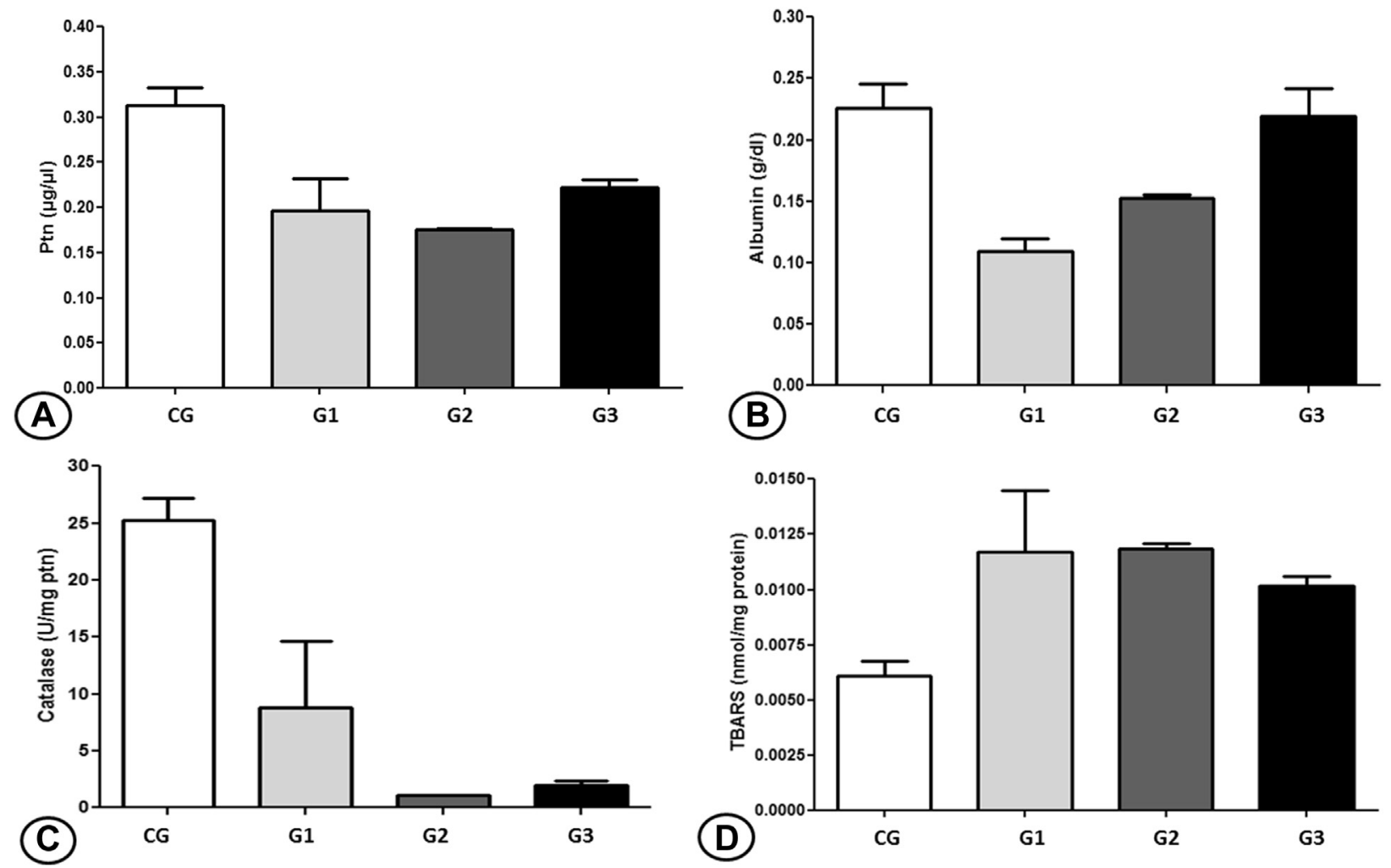

Fig. 3 - Quantification of biochemical analysis. (A) Hepatic protein levels. (B) Liver albumin levels. (C) Enzymatic activity of catalase. (D) Levels of TBARS. Group 1 (G1): studied $60 \mathrm{~d}$ after surgery; group 2 (G2): studied $90 \mathrm{~d}$ after surgery; group 3 (G3): studied $180 \mathrm{~d}$ after surgery; and group C (GC): animals without surgery.

postsurgical time, with G3 having similar concentrations as those of GC $(0.22 \pm 0.02 \times 0.11 \pm 0.01 \times 0.15 \pm 0.003 \times$ $0.22 \pm 0.02 \mathrm{~g} / \mathrm{dL} ; \mathrm{GC}, \mathrm{G} 1, \mathrm{G} 2$, and G3, respectively).

The enzyme with antioxidant function analyzed had its concentration related to the time of surgery, as the postsurgery time increased so did the catalase's activity. GC was significantly different from G1, G2, and G3 $(P<0.0001$; $25.27 \pm 1.88 \times 8.82 \pm 5.81 \times 1.09 \pm 0.04 \times 2.00 \pm 0.29 \mathrm{U} / \mathrm{mg} \mathrm{ptn} ;$ GC, G1, G2, and G3, respectively).

The oxidative stress measured through the TBARS lipid peroxidation was significantly different between the GC and G2 groups $(P=0.0381)$, with the concentration of $G 3$ being the smaller of the groups exposed to the surgical procedure $(25.27 \pm 1.88 \times 8.82 \pm 5.81 \times 1.09 \pm 0.04 \times 2.00 \pm 0.29 \mathrm{U} / \mathrm{mg} \mathrm{ptn} ;$ GC, G1, G2, and G3, respectively, Fig. 3).

\section{Discussion}

The results found suggest a good activity of the hepatic fragment placed at the rat's peritoneum. The regeneration was showed through influx of cells, necrosis, fibrosis, degeneration, neovascularization, and fibrosis, all dependent of the postsurgery time.

With its hepatic synthesis, albumin is one of the main parameters to evaluate hepatocyte activity [16]. The similar concentrations of albumin between GC and G3 infer a good capacity of synthesis by the fragment. Catalase is an intracellular enzyme found in the liver that participates in the decomposition of hydrogen peroxide, a substance closely related to oxidative stress [17]. The high concentrations of TBARS, as well as the reduced levels of catalase, show a redox unbalance in the autologous fragment.

Means of evaluating the transplanted fragment's viability as well as new models to mimic the human model have been tested. The porcine hepatic transplant from a living donor is superior to other animals methods due to its anatomic similarities with humans, but the difficulties of in situ excision of the hepatic parenchyma, among other reasons, limit its use [18]. The lack of surgical details on articles such as Katoh et al. [19] hampers this approach. Animals' availability is another difficulty, as described by Kawarasaki et al. [20], in their study with monkeys.

With the holdbacks of animal models and the own human response to transplant, autologous transplant has become an alternative. The most recent use of hepatic autologous transplant is with hard to reach tumors or tumors impossible to resect without the removal of the whole organ, but it is still functional. Hepatic surgery specialists in Beaumont remove the liver of the stricken individual and, after excision of the tumor, transplant back the remaining organ, with this fragment being responsible to regenerate itself and maintain hepatic function [10].

\section{Conclusions}

To our knowledge, this is the first study to investigate hepatic regeneration at rats' retroperitoneum. The influx of 
inflammatory cells, the parenchyma alterations, the enzymatic activities, and the redox unbalance were reduced proportionally to the postsurgical time, in addition to evidence hepatic regeneration in the hepatic fragment subject to autologous transplant in the retroperitoneum. Future studies will be necessary to determine the mechanisms responsible for the alterations found, opening new possibilities to immunologic, biochemistry, and molecular research and to potentiate the idea of biological reserves.

\section{Acknowledgment}

The authors thank the Laboratory of Experimental Nutrition (LABNEX - UFOP).

This study was financially supported by the National Council for Research and Development (CNPq) and the Federal University of Ouro Preto (UFOP).

Authors' contributions: M.F.D. contributed to the conception and design and wrote the article. M.F.D., G.M.R., and I.K.F.D.S. did the analysis and interpretation. M.F.D., T.S.B., L.F.C.P., F.G.M., and G.M.R. collected the data. S.L.S. did the approving of the final version of the article.

\section{Disclosure}

The authors reported no proprietary or commercial interest in any product mentioned or concept discussed in the article.

\section{R E F E R E N C E S}

[1] Harrison JH, Merrill JP, Murray JE. Renal homotransplantation in identical twins. Surg Forum 1956;6:432.

[2] Zhu XH, Pan JP, Wu YF, Ding YT. Establishment of a rat liver transplantation model with prolonged biliary warm ischemia time. World J Gastroenterol 2012;18:7194.

[3] Lei J, Yan L, Wang W. Donor safety in living donor liver transplantation: a single-center analysis of 300 cases. PLoS One 2013;8:e61769.
[4] Seehofer D, Eurich D, Veltzke-Schlieker W, Neuhaus P. Biliary complications after liver transplantation: old problems and new challenges. Am J Transplant 2013;13:253.

[5] Buck DG, Zajko AB. Biliary complications after orthotopic liver transplantation. Tech Vasc Interv Radiol 2008;11:51.

[6] Wojcicki M, Milkiewicz P, Silva M. Biliary tract complications after liver transplantation: a review. Dig Surg 2008;25:245.

[7] Nussler A, Konig S, Ott M, et al. Present status and perspectives of cell-based therapies for liver diseases. J Hepatol 2006;45:144.

[8] Orio F, Muscogiuri G, Palomba S, et al. Endocrinopathies after allogeneic and autologous transplantation of hematopoietic stem cells. ScientificWorldJournal 2014;2014:282147.

[9] Hata T, Uemoto S, Fujimoto Y, et al. Transplantation of engineered chimeric liver with autologous hepatocytes and xenobiotic scaffold. Ann Surg 2013;257:542.

[10] Booth C, Soker T, Baptista P, et al. Liver bioengineering: current status and future perspectives. World J Gastroenterol 2012;18:6926.

[11] Diniz MF, Dourado VA, Silva ME, et al. Cigarette smoke causes changes in liver and spleen of mice newborn exposed during pregnancy. J Cytol Histol; 2013:1.

[12] Aebi H. Catalase in vitro. Meth Enzymol 1984;105:121.

[13] Lowry OH, Rosebrough NJ, Farr AL, Randall RJ. Protein measurement with the Folin phenol reagent. J Biol Chem 1951;193:265.

[14] Draper HH, Hadley M. Malondialdehyde determination as index of lipid peroxidation. Meth Enzymol 1990;186:421.

[15] Pinnell AE, Northam BE. New automated dye-binding method for serum albumin determination with bromocresol purple. Clin Chem 1978;24:80.

[16] Saito M, Seo Y, Yano Y, et al. Serum albumin and prothrombin time before entecavir treatment in chronic hepatitis B or cirrhosis are related to amelioration of liver function after treatment. Eur J Gastroenterol Hepatol 2013;25: 1369.

[17] Gabbianelli R, Palan M, Flis DJ, et al. Imbalance in redox system of rat liver following permethrin treatment in adolescence and neonatal age. Xenobiotica 2013;43:1103.

[18] Hojo N, Ishibashi T, Yasuda T, et al. A new porcine model of autologous living-donor liver transplantation. Jichi Med Univ J; 2007:37.

[19] Katoh H, Ohkohchi N, Hirano T, et al. Viability of partial liver graft from living donor in pigs. Tohoku J Exp Med 1995;175: 179.

[20] Kawarasaki H, Iwanaka T, Tsuchida Y, et al. Partial liver transplantation from a living donor: experimental research and clinical experience. J Pediatr Surg 1994;29:518. 